\title{
多孔硅发光膜的研究
}

\author{
陈立登 季振国 马向阳 姚鸿年 阐端咪

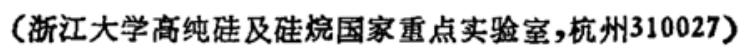

\section{关钱诃 多孔硅、光致发光、型子线阵}

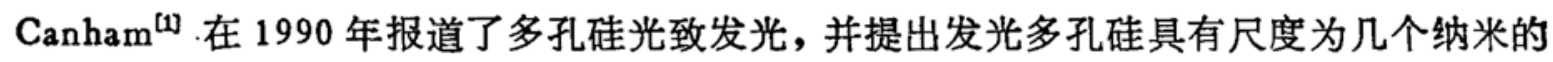
量子线阵结构. 其后不久, Richter 等制作了第一个多孔硅光发射器件 [2]. 目前, 有关多孔硅 发光的机理研究, 提出了几种设想. 多数研究者倾向于多孔硅的量子线阵结构使带隙增宽, 导 致可见苂光 ${ }^{[3]}$. 但是, 到目前为止, 发光多孔硅的量子线阵结构尚无直接的实验证据. 另外一 些观点有: 多孔硅中的硅氢烷发光 ${ }^{[a]}$; 附着在硅表面的某种分子发光 ${ }^{[1}$, 以及多孔硅表面性质 不同产生荻光 ${ }^{16}$ 等.

本文通过将发光多孔硅表面上的膜与硅祄底分离, 并采用 ESCA、红外、紫外、 $\mathrm{X}$ 射线衍 射和电镜等, 直接对分离出的膜进行了分析研究. 实验证实, 发光物质是一种含氟氢的硅氧化 合物.

\section{一、实 验}

1. 多孔硅发光膜的形成与分离实验样品为电阻率小于 $1 \Omega \cdot \mathrm{cm},\langle 111\rangle$ 晶向的 $\mathrm{p}$ 型硅 片. 样品经 $\mathrm{HNO}_{3}: \mathrm{HF}=5: 1$ 腐蚀液扡光及清洗处理. 与许多文献报道的方法相同 ${ }^{[3]}$, 硅片 作为阳极, 在 $25 \%$ 浓度的 $\mathrm{HF}$ 水溶液中电解腐蚀 $20 \mathrm{~min}$ 到 $1 \mathrm{~h}$ 后, 硅片表面形成发光多孔硅 层. 在紫外光照射下, 多孔硅层发出桔黄色荧光. 随后, 以发光多孔硅片作为阳极, 在碱性溶 液中(例如 $\mathrm{KOH}$ ) 通以电流. 在数秒钟电流冲击下, 膜即从硅片上分离出来.

分离出的膜是透明的或半透明桔黄色的薄膜, 易碎裂成粉末, 在紫外光照射下发出淡黄色 或桔黄色荧光. 荧光膜厚度与制备工艺有关. 实验观测到发光硅片上分离出苂光膜后, 硅片 不再发生荻光.

2. 苂光膜荧光谱测定使用日立 $M 850$ 荧光分光光度计分别测定了多孔硅样品及分离 出荻光膜的苂光谱, 所用的激发波长为 $250 \mathrm{~nm}$, 狭缝 $2.2 \mathrm{~nm}$. 图 1(1) 是分离前多孔硅的荧光 谱, 峰位为 $600 \mathrm{~nm}$, 谱线半峰宽约为 $140 \mathrm{~nm}$. 图 1(2) 是分离膜的荧光谱, 峰位为 $590 \mathrm{~nm}$, 谱 线半峰宽约为 $160 \mathrm{~nm}$. 两条谱线基本一致.

荻光光谱测定结果证实, 多孔硅发光与分离出的荧光膜发光来源于相同的物质.

3. X 射线衍射分析测定是在理学电机 DMAX/RA X 射线衍射仪上进行, 采用 $\mathrm{Ni}$ 滤波, Cu 辐射, 工作电压 $40 \mathrm{kV}$, 靶流 $50 \mathrm{~mA}$.

常规条件下获得的发光多孔硅,表层荧光膜较薄, 多晶衍射无法取得 $X$ 射线衍射谱图, 仅 显示硅基片的 (111)峰. 只有当荧光膜厚度超过数十微米, 才能检测到苂光膜微弱的信息. 我

1992-09-10 收稿, 1992-09-16 收修改稿 


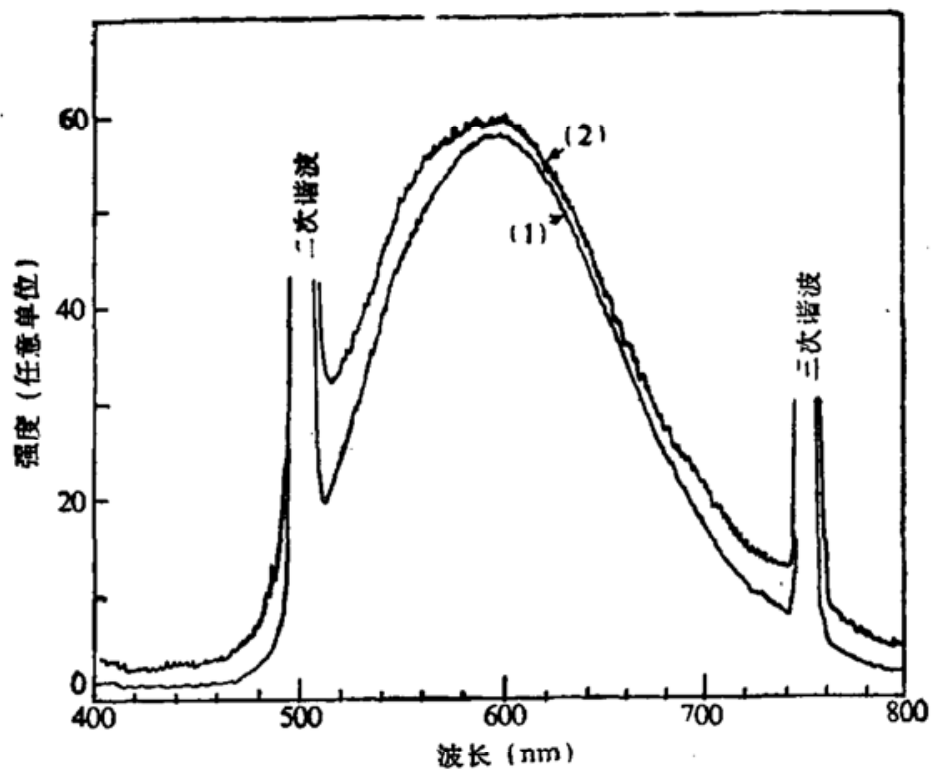

图 1 发光多孔硅(1)和分离出的荧光膜 (2)的荧光 谱

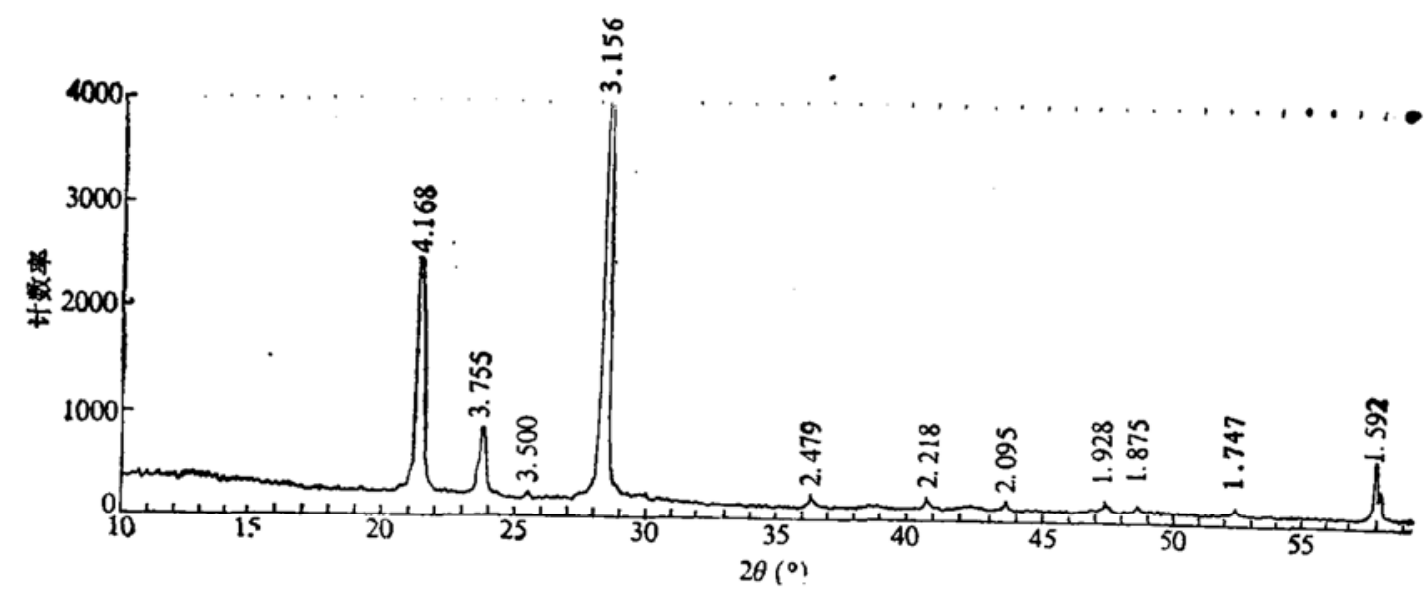

图 2 从多孔硅上分离荧光膜的 $\mathrm{x}$ 射线衍射谱

表 1 分离苂光膜的 $\mathrm{X}$ 射线衍射数据

\begin{tabular}{|c|c|c|c|c|c|}
\hline & 膜 & 射 & \multicolumn{3}{|c|}{$\alpha-\mathrm{SiO}_{2}$ JCPDS $\underset{33}{\mathrm{~N}}-1161$} \\
\hline No. & $d$ & 强度 & $d$ & (hkl) & $I$ \\
\hline 1 & 4.168 & 最强 & 4.26 & $(100)$ & 65 \\
\hline 2 & 3.755 & 次强 & 3.34 & (101) & 100 \\
\hline 3 & 3.500 & 蒻 & $\mathrm{Si}(111) \mathrm{K}_{\phi}$ & & \\
\hline 4 & 3.156 & 很强 & $S_{\mathrm{i}(111) \mathrm{K}_{a}}$ & & \\
\hline 5 & 2.479 & 䨒 & 2.46 & $(110)$ & 12 \\
\hline 6 & 2.218 & 弱 & 2.28 & $(102)$ & 12 \\
\hline 7 & 2.095 & 很鈞 & 2.12 & $(200)$ & 9 \\
\hline 8 & 1.928 & 很䨎 & $\operatorname{Si}(200) K_{a}$ & & \\
\hline 9 & 1.875 & 很的 & 1.82 & (112) & 17 \\
\hline 10 & 1.747 & 很弱 & 1.66 & (202) & 10 \\
\hline 11 & 1.592 & 次强 & 1.54 & (211) & 15 \\
\hline
\end{tabular}

第 21 谟

科学 通报 
凹从许多块样品上制取并分离出荻光膜, 用稀释的有机胶水, 粘在抛光(111)硅片上, 堆积总原 度约 $200-400 \mu \mathrm{m}$.

根据行射谱分析, 扣除硅基片(111)峰外, 其结构是比较完整的多晶体结构, 与 $\alpha$-石英相 类似,如谱线图 2 与表 1 数据.

同样我们也曾将苂光膜粘在玻璃样品架上,虽然背底较高, 但排除了（111）硅基片的影 响. 测定结果证实, 样品中桔黄色半透明荧光膜占多数时, 谱线的有一定量的 $\mathrm{Si}(111)$ 峰, 而 透明淡黄膜较多的样品, Si(111) 峰较弱, 如图 3 所示.

$X$ 射线衍射分析结果证实,分离苂光膜具有与 $\alpha$-石英相类似的多晶结构. 而且发荻光强 度较高的透明膜与发光强度较弱的桔黄色膜在组成上存在差异, 后者含有一定量的硅晶体组 分.

4. 苂光膜红外吸收谱测定 采用 NICOLET DK 型傅里叶变换红外光谱仪,测定了两 种发光强度不同的荻光膜. 为了对照, 同时也测定了 $\mathrm{P}$ 型 $\mathrm{CZ}-\mathrm{Si}$ 和 $\alpha$-石英的吸收谱线.测 试结果如图 4 所示. 发光强度较强, 发亮黄茨光的发光膜 (图 4(1)), 其红外吸收谱上未出现 $616 \mathrm{~cm}^{-1}$ 的 $\mathrm{Si}-\mathrm{Si}$ 峰. 在 $1120-480 \mathrm{~cm}^{-1}$ 区间, 与 $\alpha$-石英吸收谱线 (图 4(3)) 相比较, $\mathrm{Si}-\mathrm{O}$ 键峰很接近. 其中, $1120 \mathrm{~cm}^{-1}$ 的 $\mathrm{Si}-\mathrm{O}-\mathrm{Si}$ 键峰最强. $908.6 \mathrm{~cm}^{-1}$ 的 $\mathrm{Si}-\mathrm{H}_{2}$ 键峰和 $663 \mathrm{~cm}^{-1}$ 的 $\mathrm{Si}-\mathrm{H}$ 键峰较弱. 发暗黄荧光的苂光膜吸收谱线 (图 4(2)), 除了存在 $\mathrm{Si}-\mathrm{O}$ 键峰以外, 还存在比较强的 $616 \mathrm{~cm}^{-1}$ 的 $\mathrm{Si}-\mathrm{Si}$ 键峰以及 908 和 $663 \mathrm{~cm}^{-1}$ 的 $\mathrm{Si}-\mathrm{H}$ 键峰.
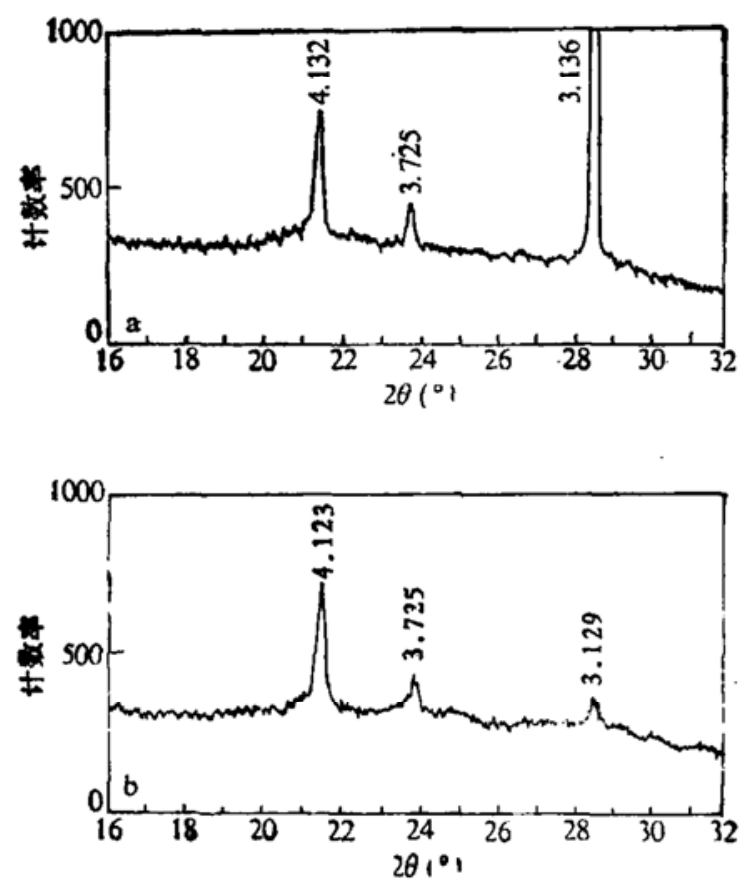

图 3 桔黄色荧光膜 (2) 与透明淡黄色荧光膜

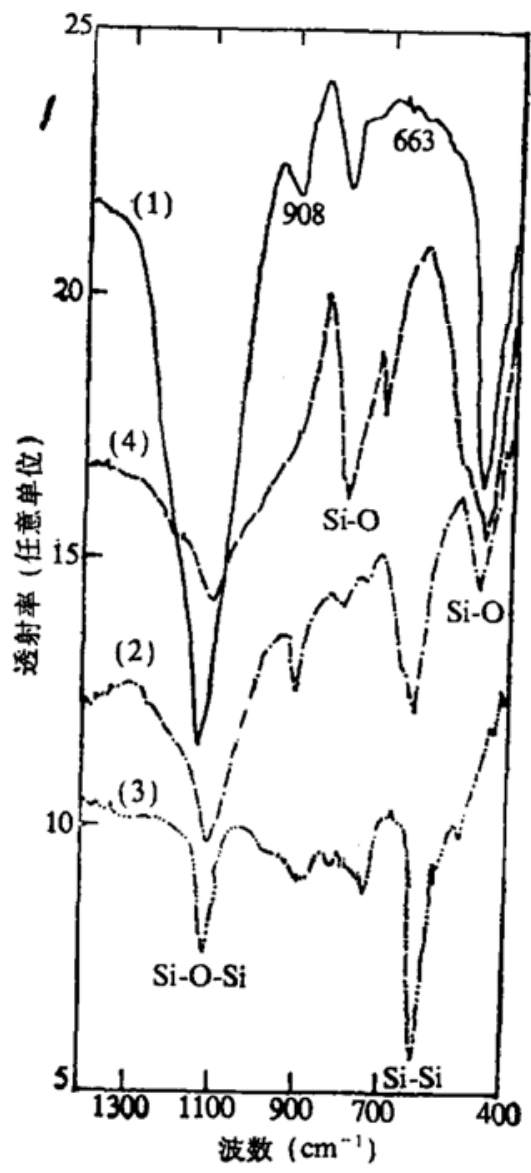

图 4 发光膜的红外光谱

(b) $\mathbf{X}$ 射线谱的比较

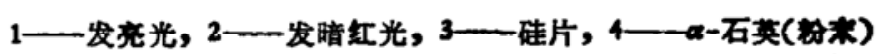




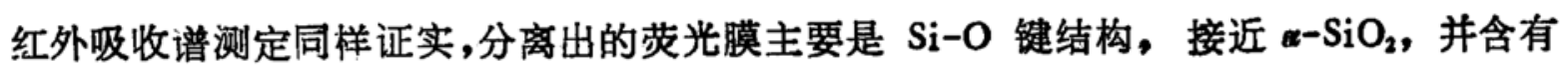
一定量的氢和晶态硅, 而茨光膜中晶态硅降低发光亮度.

5. 多孔硅苂光膜的 ESCA 研究 光电子谱测定是在 ESCALAB MK II 电子能谱仪 上进行的, 光源为 $\mathrm{Alka}$, 真空度 $\approx 5 \times 10^{-9} \mathrm{mbar}$, 用双面胶将茨光膜粘在样品台上, 共分析 了 6 个样品 ${ }^{[8]}$.

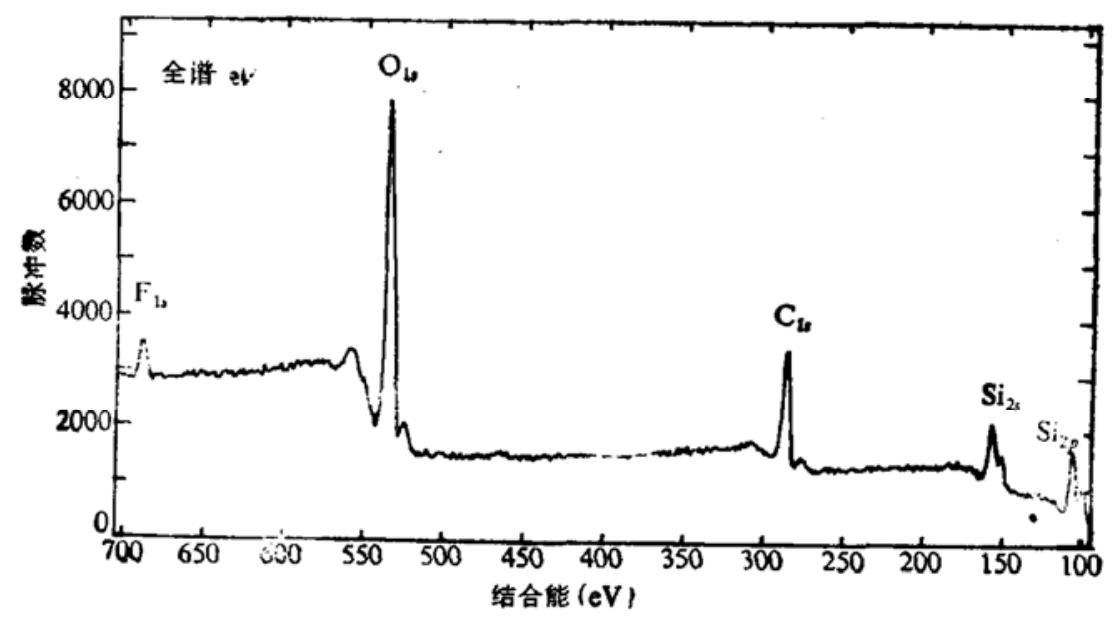

图 5 荧光膜的 $\mathrm{X}$ 光电子谱 $\left(\mathrm{AlK}_{a}\right.$ 激发)

图 5 是典型的荧光膜样品的 XPS 全谱,可以看出荧光膜特征峰的低动能端相当平坦,说 明发光膜已不是半导体而是绝缘体了. 在 XPS 灵敏度范围内我们发现样品中主要有氧、碳、 偂、硅四种元素, 其中碳主要来源于样品台等外来污染.

荧光膜中氧硅比的值在 1.45 至 1.75 之间,氟硅比的平均值约为 0.05 . 我们认为荧光膜的 主要成分为 $\mathrm{SiO}_{x}, \boldsymbol{x}$ 值低于 2 , 其结构可能与石英类似, 其中有含量约为百分之一数量级的 偂, 氟可能取代部分氧形成了硅氟氧化合物.

对于 $\mathrm{Si}_{2 p}$ 光电子峰的进一步分析指出, $\mathrm{Si}_{2 p}$ 峰包含二个部分, 其中位于 $99.8 \mathrm{eV}$ 峰对应 于晶态硅的 $2 p$ 峰; $105.7 \mathrm{eV}$ 峰对应于石英 $\mathrm{SiO}_{2}$ 中的硅 $2 p$ 峰, 图 6 是三个试样 $\mathrm{ESCA}$ 全 谱中 96-112eV 区域内 $\mathrm{Si}_{2 p}$ 光电子峰形的比较. 实验表明, 发光强度最大的荧光膜(图中曲 线 (3)), 只有 $105.7 \mathrm{eV}$ 峰, 未出现晶态硅峰; 发光强度较低的荧光膜(图中曲线 (2)), $105.7 \mathrm{eV}$ 峰减弱, 并出现 $99.8 \mathrm{eV}$ 晶态硅峰.

样品中氧的含星差别较大, 氟含量越高, 发光强度越大,晶态硅成分越多发光强度越小.因 此多。硅发光本质和硅氧化合物中的杂质有关，见表 2 .

表 2 样品中各组成的相对量 (原子\%)

\begin{tabular}{|c|c|c|c|c|c|c|}
\hline 样 品 号 & 1 & 2 & 3 & 4 & 5 & 6 \\
\hline$S_{i_{2 D}}$ (对应于 $105.7 \mathrm{eV}$ 峰) & 40.0 & 38.1 & 36.5 & 35.5 & 35.4 & 31.2 \\
\hline 0 & 56.8 & 57.1 & 58.4 & 56.6 & 57.9 & 54.0 \\
\hline $\mathbf{F}$ & 3.2 & 2.9 & 2.5 & 1.7 & 1.4 & 0.4 \\
\hline 晶态硅 (对应于 $99.8 \mathrm{eV}$ ) & 0 & 1.9 & 1.6 & 6.2 & 5.3 & 14.3 \\
\hline 荧光强度(相对值) & 1 & 0.66 & 0.21 & 0.18 & 0.09 & 0.05 \\
\hline
\end{tabular}

第 21 期

科学通报 


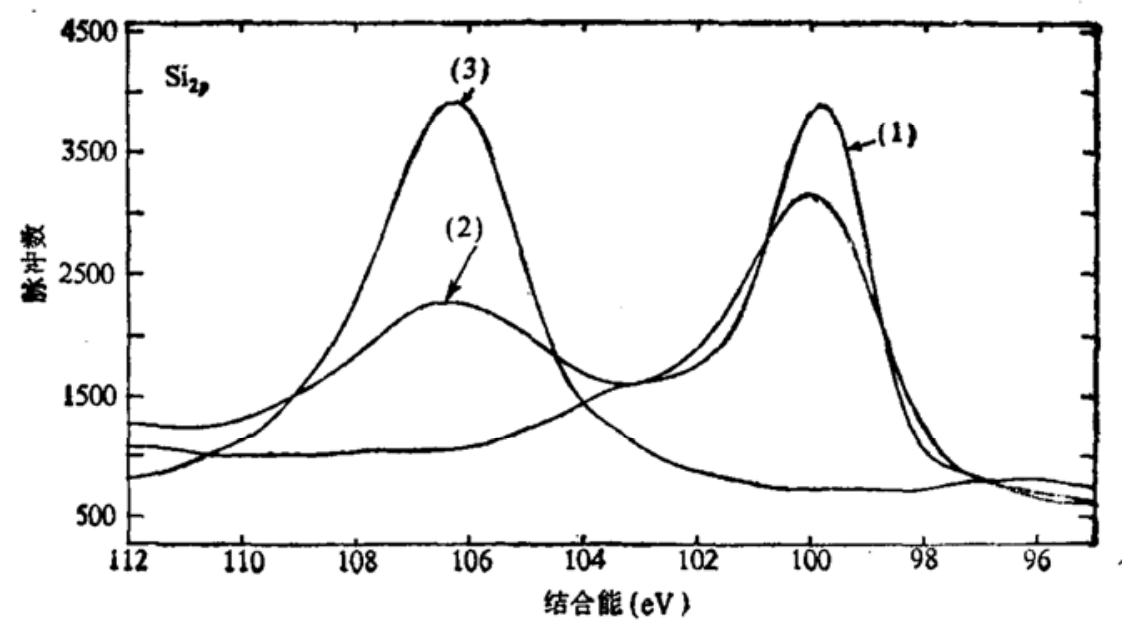

图 $6 \mathrm{Si}_{2}$, 峰的比较

\section{二、结 论}

1. 采用碱性溶液电神击方法从发光多孔硅片上成功地分离出荧光膜.

2. 用电冲击法分离取下的荧光膜, 其荧光特性与分离前的发光多孔硅一致, 实验证实多孔 碃发光是由多孔硅表层的茨光膜引起的.

3. $X$ 射线衍射、红外光谱与 $X$ 射线光电子谱测定均证实荧光膜是多相结构, 其主要组成物 是类似于 $\alpha$-石英的多晶体, 分子式为 $\mathrm{SiO}_{x}$ ( $x$ 值小于 2), 并含有少量氟氢和一定 量的晶 态硅成分.

4. 膜的发光强度与膜中氟含量有关, 氟含量越高, 发光越强; 晶态硅成分越多, 发光强度越 低。

\section{参考文献}

[1] Canham, L. T., Appl. Phys. Lett., 57 (1990), 1046.

[2] Richter, A., Steiner, P., Kozlowski, F., Lang, W., IEEE Electron. Dev. Letz., 12 (1991), 691.

[3] Koshida, N., Koyama, H., Japanese Journal of Applied Physics, 30 (1991), 1221.

[4] Tsai, C., Li, K. H., Sarathy, J. et al., Appl. Phys. Letz., 59 (1991), 28] t.

[5] Xu, Z. Y., Gal, M., Gross, M., Appl. Phys. Lett., 60 (1992), 1375.

[ 6 ] Tlschter, M. A., Collins, R. T., Stathis, J. H. et al., Appl. Phys. Lett., 60 (1992), 639. 